\title{
A Comparison of Two Prototype Laser-Optical Firing Systems
}

\author{
Gregg L. Morelli and Michelle R. Bright \\ National Nuclear Security Administration’s Kansas City Plant, 2000 E. 95 ${ }^{\text {th }}$ St., Kansas City, MO, \\ USA
}

\begin{abstract}
The design and characterization of small, ruggedized laser-optical subsystems is required for the continued development of robust laser-optical firing systems. Typically, these subsystems must be capable of generating the needed laser optical energy, delivering that energy via fiber-optical cables while taking up occupying a volume as small as possible. A novel beam splitting and fiber injection scheme has been proposed which utilizes two diffractive optical components. These components were utilized to reduce the volume of a previously designed system. A laser-optical prototype system was assembled and tested which utilized this beam splitting and fiber injection scheme along other modifications to the laser module and the power supply. This prototype was based on earlier designs that utilized environmentally proven optomechanical sub-assemblies. The system was tested to characterize the laser performance, the splitter-coupler transmission efficiency, channel-to-channel energy balance and fiber interchangeability. The results obtained for this design will be compared to the performance of a prototype system based on a more traditional beam splitting and fiber injection scheme. The traditional design utilized partially reflecting mirrors for beam splitting and plano-convex lenses for fiber injection. These results will be discussed as will their ultimate impact on future designs and packaging strategies.
\end{abstract}

Keywords: Lasers, Fiber Optical, Beam Injection, Diffractive Optical Elements, Environmentally Rugged.

\section{INTRODUCTION}

The goal of this project was to assemble and characterize a new "Diffractive" design for a fiber-coupled laser firing system and compare the results obtained to those of a "Baseline" design with proven performance. The two fibercoupled laser systems to be compared have similar laser designs, but utilize significantly different beam splitting and fiber injection designs. The two fiber-coupled laser systems will be referred to as the "Baseline System" and the "Diffractive System". The performance of these two designs was compared by evaluating a number of parameters. These included, laser output energy, beam divergence, slope efficiency, spatial intensity profile, fiber injection and splittercoupler transmission efficiency. Other factor evaluated were the ease of alignment, the required assembly time and the repeatability of the alignment process. Additional items of interest that will be commented on are the commercial availability of the diffractive optical components and the fabrication of the multi-fiber cables.

\section{BACKGROUND}

Previously, the Baseline System had been assembled and characterized ${ }^{[1]}$. At that time, nearly all of the optical and mechanical components for the Diffractive System had been received from the commercial vendors. However, due to procurement difficulties encountered on a number of the more unique components, the assembly of the Diffractive System was delayed. It has not been possible to complete the assembly and characterization of the Diffractive Systems until now. Sufficient hardware was procured to allow for the assembly and test of two prototype Diffractive Systems. The performance of the completed units will be compared to the earlier results obtained for FY07 Baseline System. Additionally, the performance variations between the two Diffractive prototypes will be examined.

The Baseline system has a laser subassembly based on a flashlamp pumped, Q-switched laser utilizing a Nd:Cr:GSGG ${ }^{[2]}$ laser rod. The multimode resonator design provides an output beam with a center weighted intensity profile appropriate for fiber injection (Figure 1). The splitter-coupler (SC) scheme is based a on straight forward approach utilizing partially reflecting mirrors, placed at 45-degree Angle of Incidence (AOI), to split the incident laser beam into three parallel channels. Three plano-convex lenses focus the beams into the individual optical fibers. Three discrete fiber-optical 
cables are each terminated with standard SMA905 connectors (Figure 2). The fibers utilized have an inner core diameter of 400 microns $(\mu \mathrm{m})$. A custom designed power supply and control electronics unit was utilized to power the laser during alignment and characterization testing. The flashlamp Pulse Forming Network (PFN) consists of a high voltage trigger circuit and an Inductor-Capacitor (LC) circuit. The flashlamp PFN circuit utilizes a 21.7 micro-farad ( $\mu$ f) capacitor and a 36 micro-henry $(\mu \mathrm{h})$ inductor to provide a critically damped pulse across the flashlamp that is approximately 80 microseconds ( $\mu \mathrm{sec}$ ) wide at the $1 / 3$ peak points. Q-switch PFN produces a high voltage $(-3 \mathrm{kV})$ pulse across the $\mathrm{LiNbO}_{3} \mathrm{Q}$-switch crystal. The high voltage pulse is flat during the laser pulse build-up time and has a fast falltime (Figure 3).

The Diffractive System has a laser subassembly that is based on a dual-flashlamp pumped, Q-switched laser utilizing a Nd:Cr:GSGG laser rod. The same multimode resonator design is utilized to provide an output beam with a center weighted intensity profile suitable for fiber injection (Figure 1).

\section{FY08 Diffractive System}



\section{FY07 Baseline System}



Figure. 1. The Diffractive and Baseline system, flashlamp pumped, Q-Switched, Nd:Cr:GSGG laser.

The SC scheme employed in this system takes a more novel approach. A single plano-convex lens and two special designed diffractive optical components are utilized to separate the incident laser beam into three very closely spaced beams and focus them into optical fibers. A custom designed fiber optical connector assembly accurately holds and orients the three $365 \mu \mathrm{m}$ diameter fibers (Figure 2). 
FY08 Diffractive

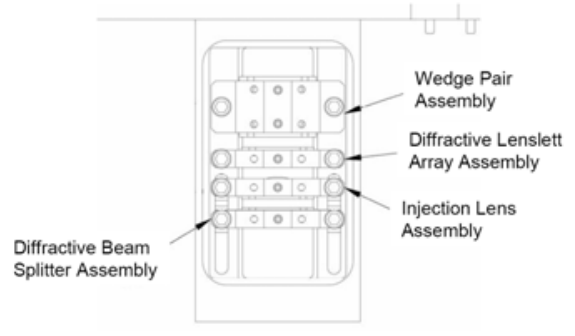

FY07 Baseline



Figure. 2. The splitter-coupler modules for the Diffractive and Baseline Systems respectively. 
Power supply design utilized to fire the laser during alignment and characterization testing was modified to allow more rapid pumping of the laser rod. The flashlamp PFN circuit utilizes a $9.2 \mu \mathrm{f}$ capacitor without an inductor to provide a pulse across the flashlamp that is approximately $18 \mu$ sec wide at the $1 / 3$ peak points. To avoid flashlamp failures associated with the high-current densities generated, the two lamps are connected in series. A commercially available Qswitch driver was utilized to produces a high voltage $(-3 \mathrm{kV})$ pulse across the $\mathrm{LiNbO}_{3} \mathrm{Q}$-switch crystal. The high voltage pulse is flat during the laser pulse build-up time and has a fast fall-time (Figure 3).

\section{FY08 Diffractive System}

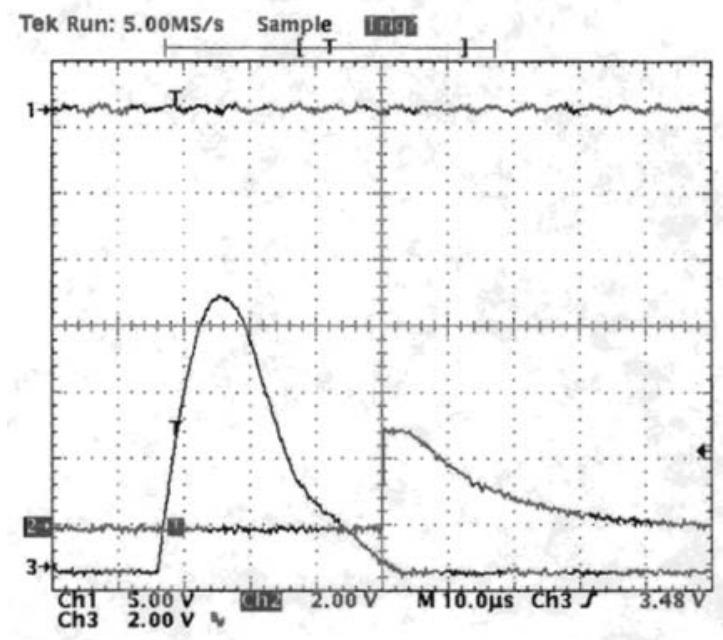

FY07 Baseline System



Figure. 3. The flashlamp and Q-switch waveforms generated by the power supplies utilized for the Diffractive and Baseline Systems respectively.

\section{SYSTEM PERFORMANCE COMPARISON}

\subsection{Laser Module Performance}

A typical operating point for the Baseline System's laser would consist of obtaining approximately 68 milli-Joules (mJ) of output energy for an electrical input energy of 7.8 Joules. The calculated slope efficiency, laser output energy to stored electrical energy, would be approximately $1.4 \%$ with a point efficiency of approximately $0.87 \%$ (Figure 4 ). The optical output would have a 12.5 nanoseconds (ns) pulsewidth, measured at the Full Width Half Maximum (FWHM) position.

Based on the similarities between the two laser designs, it was anticipated that the Diffactive System's dual-flashlamp laser would perform in much the same manner as the Baseline laser. A goal of obtaining between $65 \mathrm{~mJ}$ and $80 \mathrm{~mJ}$ of laser output energy with a 10ns to 12ns pulsewidth, measured at FWHM, was set. It seemed reasonable to expect that many of the other parameters would also be the same or similar, including the beam diameter, the temporal and spatial profiles.

As the comparison of the Diffractive System's laser performance began it became obvious very quickly that the power supply electronics designed to facilitate a more rapid pumping of the laser rod, were not effectively driving the laser. The optical energy generated by the dual-flashlamp assembly, utilizing the modified power supply, was not producing the needed laser output energy or pulsewidth. The laser energy was low, less that $20 \mathrm{~mJ}$ of output for an input energy of 13.8J, a point efficiency of only $0.14 \%$. Additionally, the optical pulsewidths obtained were too long, measuring between $14 \mathrm{~ns}$ and $30 \mathrm{~ns}$. The $9.2 \mu \mathrm{f}$ power supply was abandoned at this point. Laser module comparisons and subsequent System comparisons will be based on results obtained utilizing the Baseline power supply configured with a $21.7 \mu \mathrm{f}$ capacitor and $36 \mu \mathrm{h}$ inductor. 



Figure. 4. Baseline System - Laser slope efficiency and pulsewidth plots as a function of electrical input energy.

Two prototype laser modules were assembled and characterized, Serial Numbers 1001 and 1002. The lasers performed markedly different having a number of interesting variations. An operating point for SN 1001 was selected which produced approximately $69.5 \mathrm{~mJ}$ of output energy for an electrical input energy of 20.3J. A slope efficiency of approximately $0.8 \%$ was obtained with a point efficiency of $0.34 \%$ (Figure 5 ). The pulsewidth was $11.5 \mathrm{~ns}$, measured at the FWHM position. An operating point for SN 1002 was selected which produced approximately 65.3mJ of output energy for an electrical input energy of 23.2J. A slope efficiency of approximately $0.4 \%$ was obtained with a point efficiency of $0.28 \%$ (Figure 5). The pulsewidth was 13.9ns, measured at the FWHM position. The output of the Diffractive laser and the Baseline laser both had a smooth temporal profile with very little modulation (Figure 6).


Figure. 5. Diffractive Systems - Laser slope efficiency and pulsewidth plots for Serial Numbers 1001 and 1002. 
FY08 Diffractive System

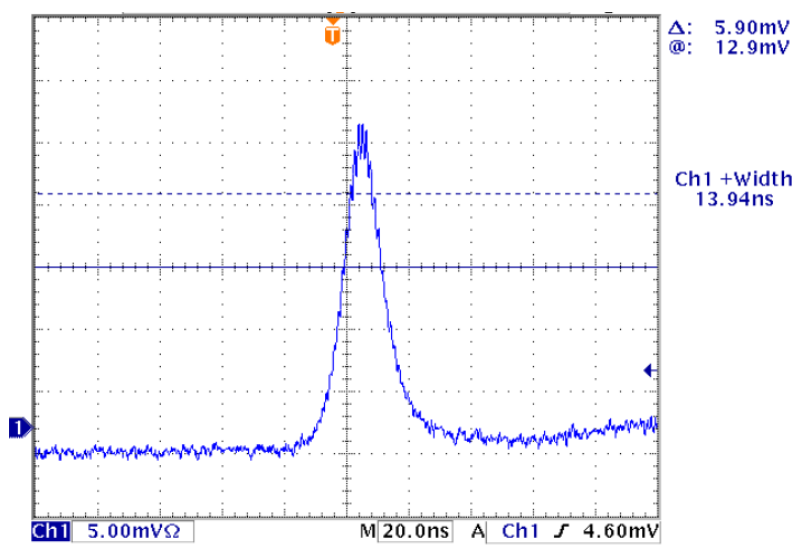

FY07 Baseline System



Figure. 6. Pulsewidth waveform plots for Diffractive and Baseline Systems respectively.

The Baseline System's laser provided a far-field spatial intensity profile that is smooth and symmetric with a center weighted intensity pattern (Figure 7). The full angle, far-field beam divergence is 2.0 milli-radians (mR), when measured at the $90 \%$ points. This far-field profile is typical of multimode lasers utilizing this type of Polarization Output Coupled (POC) resonator design.



Figure. 7. Baseline System’s laser far-field, spatial intensity profile.

The Diffractive System's laser provided a far-field spatial intensity profile that was symmetric, but was not as smooth as the Baseline laser. Additionally, both of the Diffractive System prototypes exhibited an intensity profile with a dip in the center (Figure 8). While the far-field beam divergence was approximately 2.0 milli-radians (mR) for both prototypes, qualitatively the two beams were noticeable different. Serial number 1001 had a more pronounced dip in the center of the intensity profile. Even with this on-axis "hole" in the intensity profile, overall the beam was more intense in the center than serial number 1002. Serial number 1002 did not have as pronounced of an on-axis "hole" in the intensity profile and the beam was more uniform at the edges. 



Figure. 8. Diffractive System’s laser far-field spatial intensity profiles.

\subsection{Splitter-Coupler Module Performance}

The Baseline System's SC module is capable of providing an energy split ratio, between the three channels, that is routinely within $\pm 3 \%$. This is due to the fact that the Baseline SC design utilizes half wavelplates to compensate for reflectivity variations in the dielectric coated mirrors.

The Diffractive System's SC design has no similar capability that allows for the active balancing of the energy distribution between the channels. The design of the grating beamsplitter pre-determines the amount of incident energy in the channels. The grating beamsplitter was designed with the goal of equally balancing and maximizing the amount of transmitted energy in the three central spots. In reality there is a nontrivial amount of energy diffracted into Channels 4 and 5 (Figure 9).



Figure. 9. The spatial intensity pattern generated by the diffractive beamsplitter.

Transmission measurements performed on the diffractive grating revealed that approximately $3 \%$ of the incident laser energy is contained in each of the outer most channels (Table 1). Initially this may seem like an insignificant amount of stray energy to be concerned about, however past experience has shown that even small amounts of Q-switched laser energy can be a problem. When $0.5 \mathrm{~mJ}$ of laser energy, with a pulsewidth of $12.5 \mathrm{~ns}$ is focused down to a spot $290 \mu \mathrm{m}$ in diameter, a peak power density of $120 \mathrm{Mega}-\mathrm{Watts} / \mathrm{cm}^{2}$ can be generated. This is more than sufficient to damage the surface of a stainless steel SMA 905 fiber optic connector ferrule. If the ablated material happens to land on the polished fiber face, the next shot from the injection laser will most certainly damage the fiber. 
Table. 1. Diffractive Splitter-Coupler’s Normalized Channel-to-Channel Energy Variations.

\begin{tabular}{|c|c|c|}
\hline \multicolumn{3}{|c|}{ Diffractive Beamsplitter Normalized } \\
Transmission
\end{tabular}

To address the issue of residual energy in the higher diffraction orders, a mask was designed and fabricated out of Schott KG3 filter glass. The mask has an oblong slot which that allows the three middle beams to pass though without being attenuated, while blocking the outer two beams (Figure 10).
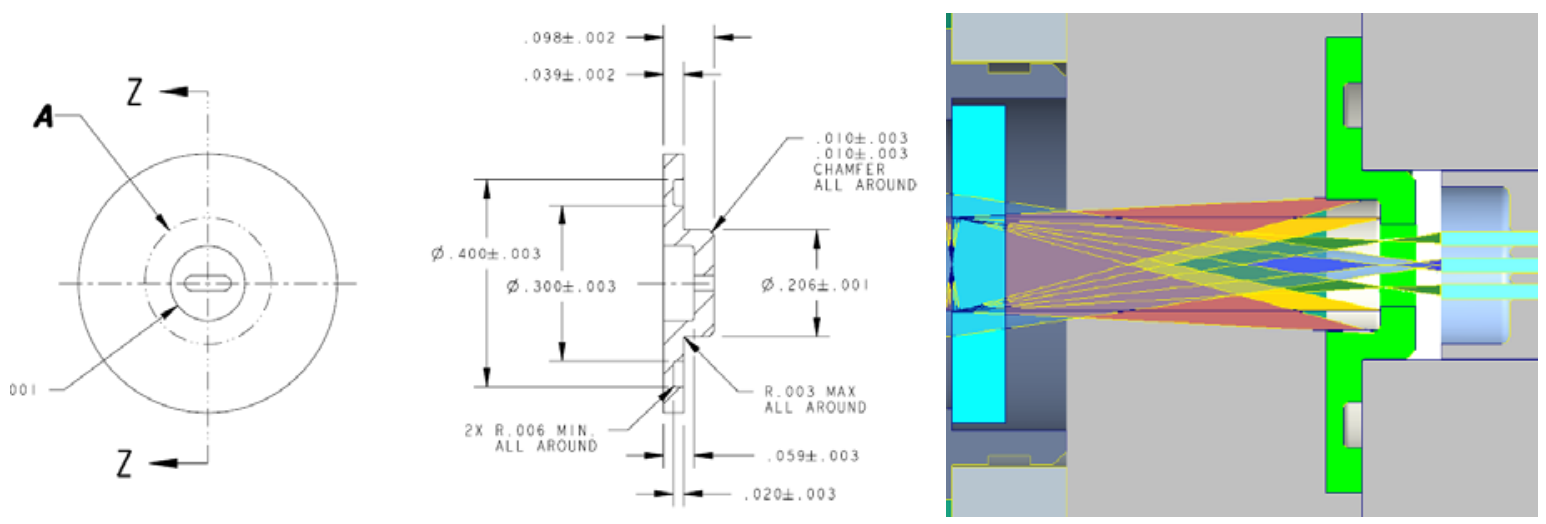

Figure. 10. The KG3 mask utilized in the Diffractive SC modules.

The KG3 filter glass absorbs the stray laser energy diffracted into spots 4 and 5 . The following measurements and calculation were performed only considering the three middle spots. Given the fact that the Diffractive System's SC does not have the capability to actively balance the energy distribution between these channels, it had been anticipated that the energy split ratio between them would be within $\pm 5 \%$. This estimate was based on the design criteria for the diffractive beamsplitter. It was also anticipated that the on-axis beam would have slightly more energy that the two outer beams. Testing of the Diffractive SC revealed that the three channels were not equally balanced. The middle beam, Channel 2, as expected was consistently higher in transmitted energy that the other two channels (Table 2).

Table. 2. Diffractive Splitter-Coupler Normalized Split Ratio Percentages.

\begin{tabular}{|l|c|l|c|}
\hline \multicolumn{3}{|c|}{ Normalized Split Ratio Percentages } \\
\hline \multicolumn{2}{|c|}{ Serial No. 1001 } & \multicolumn{2}{c|}{ Serial No. 1002 } \\
\hline Channel 1 & $30.6 \%$ & Channel 1 & $33.0 \%$ \\
\hline Channel 2 & $38.9 \%$ & Channel 2 & $37.7 \%$ \\
\hline Channel 3 & $30.5 \%$ & Channel 3 & $29.3 \%$ \\
\hline
\end{tabular}


Some interesting performances variations between the two prototype SC modules were observed. For Serial Number 1001, Channel Number 2 had approximately 5.6\% more transmitted energy than desired. Channels 1 and 3 had 2.7\% and $2.8 \%$ less energy than desired respectively. Although low in energy, these channels were equal to each other to within $0.1 \%$. It has been suggested that this is an indicator of how well aligned the incident laser beam is to the surface normal of the diffractive beamsplitter. For Serial Number 1002, Channel Number 2 had approximately 4.3\% more transmitted energy than desired. Channels 1 and 3 had $0.3 \%$ and $4.0 \%$ less energy than desired respectively. The larger energy difference between these two channels, 3.7\%, indicates a larger amount of angular misaligned between the incident laser beam and the diffractive beamsplitter (Table 3).

Table. 3. Diffractive Splitter-Coupler Normalized Channel-to-Channel Energy Variations.

\begin{tabular}{|l|c|c|}
\hline \multicolumn{3}{|c|}{ Channel-to-Channel Energy Variations } \\
\hline & Serial No. 1001 & Serial No. 1002 \\
\hline Channel 1 to Channel 3 & $0.1 \%$ & $3.7 \%$ \\
\hline Channel 2 to Channel 1 & $8.3 \%$ & $4.7 \%$ \\
\hline Channel 2 to Channel 3 & $8.4 \%$ & $8.4 \%$ \\
\hline & & \\
\hline CH 1 to Design Goal (33.3\%) & $-2.7 \%$ & $-0.3 \%$ \\
\hline CH 2 to Design Goal (33.3\%) & $5.6 \%$ & $4.3 \%$ \\
\hline CH 3 to Design Goal (33.3\%) & $-2.8 \%$ & $-4.0 \%$ \\
\hline
\end{tabular}

The overall transmission efficiency for the Baseline SC design is typically very good. Measured values of between 90$92 \%$ are common. It had been anticipated that the overall transmission efficiency of Diffractive SC design would be less than that achieved with the Baseline design. It was expected that the diffractive optical components would have more scattering losses than standard dielectric coatings. The transmission efficiency for Diffractive SC design was measured at approximately $90 \%$. The performance of the two prototypes was similar with Serial Number 1001 having a slightly lower value than Serial Number 1002.

The focusing and fiber injection characteristics of the Baseline SC design are fairly well understood and predictable. The laser's beam divergence and the focal length of the injection lens determine spot diameter achievable. It had been anticipated that the focusing and fiber injection characteristics of the Diffractive SC design would be similar. During the assemble and test of Diffractive SC Serial Number 1001 difficulty was encounter while attempting to focus the beams down to a spot $290 \mu \mathrm{m}$ in diameter corresponding to the desired $80 \%$ fill factor for the $365 \mu \mathrm{m}$ fiber. The smallest spot achievable was actually $365 \mu \mathrm{m}$ in diameter. This spot size encircled approximately $95 \%$ of the total energy in the beam. It was ultimately decides to move forward and to complete the planned test and characterization with the lenslet array installed.

During the assemble and test of Diffractive SC Serial Number 1002 difficulty was encounter again while attempting to focus the beams down to a spot $290 \mu \mathrm{m}$ in diameter. This time however the smallest spot achievable was $360 \mu \mathrm{m}$ in diameter and only $93 \%$ of the total energy was encircle. This time the decision was made to remove the lenslet array and attempt to re-focus the beams. Once the lenslet array was removed from the optical path, the beams could easily be focused down to spots that were very small in diameter. It was possible to focus the beams down to spots that were less than $200 \mu \mathrm{m}$ in diameter. The beams were focused to a spot $290 \mu \mathrm{m}$ in diameter. This spot diameter encircled approximately $97 \%$ of the total energy in the beam. It was decided that Diffractive SC Serial Number 1002 would be aligned and characterized without installing the lenslet array. 
The Baseline SC design is capable of injecting a maximum energy of between 40 and $45 \mathrm{~mJ}$, a fluence of approximately $56 \mathrm{~J} / \mathrm{cm}^{2}$, into each of the $400 \mu \mathrm{m}$ fibers. A focused spot diameter that was approximately $80 \%$ of the fiber's core diameter was utilized. The SC was operated at an energy level of $25 \mathrm{~mJ}$ per channel and an injection spot diameter $320 \mu \mathrm{m}$. Operating at this fluence level, approximately $31 \mathrm{~J} / \mathrm{cm}^{2}$, laser damage is seldom a problem. This assumes injection into a clean, well aligned optical fiber.

Laser damage related issues were not expected to be a problem for the Diffractive SC design, as long as the beams could be well aligned to the multi-fiber cable. There had been some initial concern about the smaller diameter fibers. A fiber core diameter of $365 \mu \mathrm{m}$ meant that an injection spot diameter of approximately $290 \mu \mathrm{m}$ was needed. The increase in fluence, due to the smaller injection diameter, was expected to be offset by presence of the diffractive lenslet array. The diffractive lenslet array acts to smooth the laser beam's spatial intensity profile and eliminate "hot spots" in the beam ${ }^{[3]}$. The Diffractive SC was operated at a maximum energy level of $20.4 \mathrm{~mJ}$, a fluence of $30.5 \mathrm{~J} / \mathrm{cm}^{2}$ (Table 4). No laser damage related issues were encountered, since the operating point had been chosen to provide adequate margin. The successful operation of the Diffractive SC at this energy level was considered a good indication of the alignment accuracy achieved.

Table. 4. Diffractive Splitter-Coupler Energy Measurements.

\begin{tabular}{|c|c|c|c|c|c|}
\hline \multicolumn{6}{|c|}{ Energy Measurements Through Fibers } \\
\hline \multicolumn{3}{|c|}{ Serial No. 1001} & \multicolumn{3}{|c|}{ Serial No. 1002} \\
\hline & Energy (mJ) & Fluence $\left(\mathrm{J} / \mathrm{cm}^{2}\right)$ & & Energy (mJ) & Fluence $\left(\mathrm{J} / \mathrm{cm}^{2}\right)$ \\
\hline Channel 1 & 16.2 & 24.2 & Channel 1 & 16.8 & 25.1 \\
\hline Channel 2 & 20.4 & 30.5 & Channel 2 & 20 & 29.9 \\
\hline Channel 3 & 16.9 & 25.2 & Channel 3 & 16.5 & 24.7 \\
\hline
\end{tabular}

Air breakdown and plasma formation at the intermediate focal plane of the injection lens has seldom been a problem for the Baseline SC design and it was not expected that it would be a problem for the Diffractive design. It was anticipated that utilizing the lenslet array in this design would also increasing the margin for air breakdown. During the testing and characterization of the Diffractive SC prototypes air breakdown was not observed.

Past experience with the Baseline SC design has demonstrated that the interchangeability of fiber-optical cables is typically very good. It had been anticipated that the fiber-optical cable interchangeability for the Diffractive SC would be similar. The smaller diameter fibers in the multi-fiber connector were a concern but the keyed feature of the connector was expected locate the fibers with improved repeatability. During the testing and characterization of the Diffractive SC prototypes, interchanging the multi-fiber cable did produce any adverse results. 
The Baseline SC design produces a nice spatial intensity profiles at the exit faces of the $400 \mu \mathrm{m}$ diameter fibers. The intensity profiles are center weighted and relatively smooth with a slight peak-to-average variations across the faces (Figure 11). Performance variations with cable length and fiber core diameter have been observed.



Figure. 11. Baseline System- Spatial Intensity Profile from exit faces of 400micron diameter optical fibers.

It had been anticipated that Diffractive SC design would provide spatial intensity profiles that were just as smooth as those obtained from the Baseline design. The Diffractive SC design utilizes step index, multi-mode fiber with an inner core diameter of $365 \mu \mathrm{m}$. It was not expected the slight decrease in fiber diameter would appreciable modify the spatial intensity profiles obtained. The spatial intensity profiles obtained from tests performed using the Diffractive SC prototypes we quite good (Figure 12).



Figure. 12. Diffractive Splitter-Coupler - Spatial Intensity Profiles from exit faces of 365micron diameter optical fibers.

\subsection{Splitter-Coupler Alignment}

The alignment procedure developed and utilized for the Baseline S/C design has been proven to be accurate and repeatable ${ }^{[4]}$. It had been anticipate that the alignment procedure required for the Diffractive S/C design would be similar in many ways to that of the Baseline S/C. However, it was expected that a larger number of iterations would be required to successfully complete the alignment. The limited amount of past experience gained from working with diffractive SC prototypes, had highlighted the difficulty involved in simultaneously optimizing all the channels at once. The anticipated performance was therefore not expected to be as good as the Baseline S/C.

The alignment procedure utilized for the Diffractive S/C was initially very similar to that utilized for the Baseline S/C. All of the familiar tools were utilized. A laser intensity profiling system, a coherent fiber bundle terminated with the same custom designed, multi-fiber connector and a CW, Nd:YAG alignment laser. The same process was also utilized, 
starting with the CW laser and the coherent bundle, the grating beamsplitter was rotated to orient the three spots along a horizontal line. The rotating wedge pair was utilized to align the middle beam to the coherent bundle in the same location as the center fiber in the multi fiber connector. The grating beamsplitter was translated to adjust the spot-to-spot spacing until the outer two spots were placed in the correct location. The pulsed laser was utilized to accurately set the diameter of the focused beams on the input face of the optical fibers. Once the spot diameters were set, re-adjustments of the rotating wedge pair and the grating beamsplitter were needed. As expected, a number of iterations were then required to improve the alignment. At this point, rather than continuing to iterate, the standard procedure was supplemented with a number of modification which appeared to shorten the process.

A microscope with image capture and measurement software, a "V-block" to hold the multi-fiber connector in a repeatable manner, small circular pieces of Zap-It alignment paper and double-stick tape were utilized. The small pieces of Zap-It alignment paper were taped onto a surrogate multi-fiber connector having the same fiber-to-fiber spacing. The laser was fired at the connector and the beam pattern was transferred to the Zap-It paper. The connector was removed and measurements were taken under the microscope. Based on these measurements, the needed adjustments to the rotating wedge pair and/or the grating beamsplitter were determined. This method once perfected provided the most reliable alignment information and reduced the number of iterations required to successfully complete the process.

\section{SUMMARY}

The characterization of a promising new design for a fiber-coupled laser firing systems was completed. The new system utilizes a novel diffractive SC design. The new system's performance results were compared and contrasted to that of the Baseline system. The performance of the two laser designs was comparable in a number of areas and significantly different is others. The amount of time required for laser alignment, the output energy, pulsewidth, and beam divergence were similar for both designs. While the slope efficiency and the far field spatial intensity profiles were very different. The performance of the two SC designs was also comparable in a number of areas and significantly different is others. The accuracy and repeatability of the alignment process, the overall transmission efficiency, and the spatial intensity profiles at the fiber exit faces were all somewhat similar. Additionally, for the limited range of tests performed, the resistance to laser damage and air breakdown was similar. While the amount of time required for SC alignment, the energy split ratios and the fiber injection characteristics were very different. The Diffractive System's SC required about twice as much time and effort to align as the Baseline System's SC. It was possible to deal with some of the alignment difficulty encountered utilizing modifications to the standard alignment procedure.

Other items worth commenting on are the cost and availability of the diffractive optical components. In small quantities these optics continue to be very expense and have long lead-times. The multi-fiber cables utilized for this work were custom made at Sandia National Laboratories, since a commercial source for cables does not exist. The cables proved to be extremely difficult and time consuming to fabricate. The yield for the first batch was very low. Of the five cables started, only one ultimately proved useable. The inability of the $9.2 \mu \mathrm{f}$ power supply to effectively driving the laser is an area open to further investigation.

\section{FUTURE WORK}

An initial plan to complete two additional Diffractive Systems has been put on hold as Sandia National Laboratory Engineers undertake a number of re-design activities. Near term plans to support environmental testing with a redesigned system are in the final planning stages. These tests would incorporate packaged electronics modules, currently being designed and assembled at Sandia. Longer term plans are still concerned with developing a well defined strategy to hermetically seal the entire laser-optical system. A combined approach that would make use of sealed glass windows, hermetic fiber-optical connectors, welded electrical feed-throughs and covers is still consider the best strategy. 


\section{ACKNOWLEDGMENTS}

This work was supported by the U.S. Department of Energy under contract DE-AC04-76-DP00613. Additional project specific funding was provided by Sandia National Laboratories. The authors would like to recognize and thank Tanner Vaughn for his work in the laboratory bonding, aligning and characterizing the numerous optical assemblies, lasers and SC systems. Additionally, Dante Berry of Sandia National Laboratories must be recognized for his painstaking efforts at fabricating the multi-fiber cables.

This work was the result of a highly collaborative relationship between Scientists and Engineers at Sandia National Laboratory and the Kansas City Plant. The conceptual design activity, while primarily the responsibility the National Laboratory, was preformed with the considerable involvement of the production agency. This "Concurrent Engineering" model has proven to be a very effective method for reducing the development cycle time required for these prototype systems.

\section{REFERENCES}

[1] G. Morelli, "Design and Assembly Strategies for Two Laser-Optical Firing Systems", Proceedings of SPIE Vol.6662, Aug. 2007.

[2] W. Koechner, Solid-State Laser Engineering, $5^{\text {th }}$ Edition, Springer-Verlag Berlin Heidlberg, pp. pp. 57, 1999.

[3] L. Weichman, "DOI Fiber Injection System-Lenslet Array \& Injection Lens”, AFDS Technical Presentation, Sandia National Laboratory, 1999.

[4] W. Koechner, Solid-State Laser Engineering, $5^{\text {th }}$ Edition, Springer-Verlag Berlin Heidlberg, pp. 233-235, 1999. 